\title{
Design of High Efficiency X - ray Detector
}

\author{
Yang Bo \\ School of Electronic \& Information Engineering, Foshan University \\ Foshan, Guangdong, China \\ Phone: +86-0757-829-81728, Fax: +86-0757-829-81633, Email: fsttww@yeah.net
}

\begin{abstract}
This paper presents a fast time response, high efficiency miniaturized $X$ ray detector design.Mainly includes the front readout circuit and signal processing circuit, is composed of plastic scintillator, photomultiplier tubes, high-performance precharge amplifier circuit..For $x$-ray detector at the field site power requirements, design a high efficiency and low ripple high voltage power supply.The selection principle of the scintillation and the multiplier is given. The system has the advantages of high speed, high resolution, high reliability and so on.The experimental results show that the $X$ - ray detector system can meet the requirement of high resolution and low noise, and the response time of the detector is up to 56ps.
\end{abstract}

Keywords-X-ray, detector, plastic scintillator, photomultiplier tube, high voltage supplier

\section{INTRODUCTION}

Measuring projectile muzzle velocity has the important theory significance and the practical significance for internal and external ballistics.According to the determination of the bore pressure curves and the size of the projectile muzzle velocity, can be analyzed to study the gunpowder combustion ruly from inside the bore and the influence of all kinds of work.In terms of external ballistics, it is one of the original data for researching the law of the projectile flying in the air and calculating artillery firing range. Usually exterior ballistics speed test system is mainly laser target or Doppler radar velocity- measuring method.The Laser test method is simple, but the intermediate ballistic trajectory parameters testing in specific environment, the laser can not pass through the gunpowder gas ejected from the bore,on the other hand due to the gunpowder gas light, the photosensitive element makes it difficult to distinguish with the laser off, easy to produce false triggering. Although the Doppler radar velocity measurement method is advanced, but the muzzle ionization zone will make the measurement accuracy is greatly reduced, can not reach the expected effect for the test of the muzzle velocity of projectile. X-ray is an electromagnetic wave, the wavelength is very short, mostly in the range between $0.001 \mathrm{~mm} \sim 10 \mathrm{~mm}$, This is electromagnetic radiation having a wavelength between ultraviolet rays and $\gamma$-rays .X rays are usually electrically neutral, which is characterized by high stability, even in the electric field and magnetic field is not easy to deflection.X ray testing technique is a nondestructive, non-contact advanced measurement technology, $\mathrm{X}$ - ray itself has a strong penetrating ability, is not affected by the muzzle ionization zone.The zone-block target for testing system is simple, high sensitivity, etc., The $\mathrm{X}$-ray testing technology combined with the zone-block target, forming a new test method of the intermediate ballistic parameters. This paper is aimed at the requirements of the short pulse $\mathrm{X}$ ray detector design, combined with the specific measurement environment for analysis and research, and ultimately determine a fast time response $\mathrm{X}$ - ray detection system based on the scintillation detection method.On the $\mathrm{x}$ ray detector involved scintillator, photomultiplier signal amplification circuit and other components and circuits have been analyzed and studied,The basic principle is given for the choice of components and circuit in the design. The conclusion of the analysis and design has guiding significance for the design of related $\mathrm{X}$ - ray detection.

\section{DETECTOR DESIGN}

The single target detection circuit consists of scintillation crystal ,photomultiplier tubes,current amplifier, current-tovoltage converter and integrator, the X-ray source detected principle diagram on single target is shown in Fig. 1.The output pulse of nuclear radiation detectors, formed after amplifying, output to the integrator, the integrator output a voltage proportional to the input pulse rate, this voltage to trigger, the trigger has a " 0 " and "1" two states to reflect the signal changes, so as to control the subsequent circuit, before discussing the design of the minimum current value of the Xray source,, first define some notation:

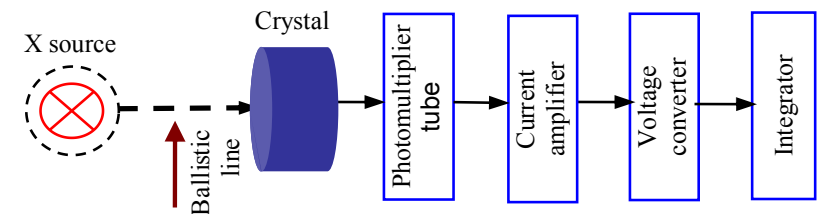

Figure 1. The X-ray source detected principle diagram on single target

There are many different kinds of nuclear radiation detector and Usually meet with gas ionization detector, scintillation detector, semiconductor detector, etc.But because the scintillation detector has the advantages of high efficiency, short response time, and detected radiation energy energy, etc., and is widely used in X-ray detection. Based on this, the scintillation detector is chosen in this problem. The scintillation detector consists of a scintillator and photomultiplier tubes and other components (see Figure 2), their work can be divided into five interrelated aspects.(1) The radiation interacts with the scintillator causing loss of energy, and make the atoms, molecules of the scintillator produce ionization and excitation. (2) In the scintillator excited states of atoms and molecules emit photons in the deexcitation process.(3)Using the reflector 
and the photoconductance, the photons are collected as far as possible on the photocathode of photomultiplier tubes . Due to the photoelectric effect, the photons can hit photoelectrons on the photocathode:(4) Photoelectron in the photomultiplier tube, multiplied through all multiplication stages, the electrons number can be increased several orders of magnitude, through the multiplied electron beam generates an electrical signal on the anode load.(5)This signal is recorded by the circuit and generates a voltage pulse across the load resistor, the output pulse amplitude is about several hundred millivolts to several volts. Under certain conditions, the output pulse amplitude is proportional to the energy of incident radiation lost in the scintillator.From the above process, how to choose the scintillator and photomultiplier tubes with the excellent performance, how to improve the collection efficiency of photons, are very important, following the main part of the scintillation detectors - scintillator and photomultiplier tubes were discussed .Next, the main part of the scintillation detectors- scintillator and photomultiplier tubes are discussed respectively.

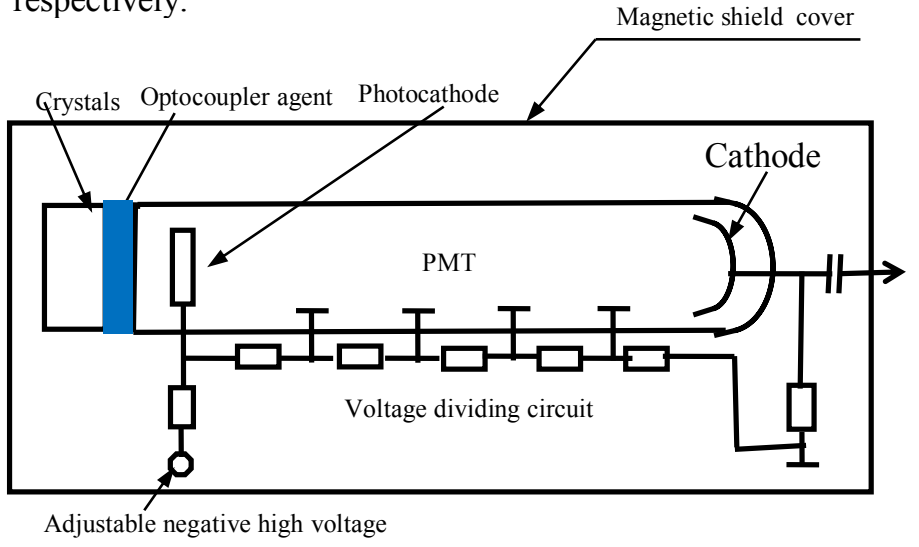

Figure 2. The structure diagram of scintillation detector

\section{A. Selection of crystal (scintillation)}

Material capable of emitting light Irradiated by radiation are collectively referred to as "scintillation" or "phosphor",this is due to the radiation particles through material will make the atoms or molecules excited, be excited atoms or molecules from the excited state to the ground state will put out,this is visible light.There are many types of scintillator, according to the state of matter are solid, liquid, gas. According to the chemical composition can be divided into two categories, inorganic scintillator, organic scintillator, commonly used some of the scintillator as shown in table I.The main physical characteristics of the scintillator has the following three key indicators:

1) Luminous efficiency, or conversion power. It is the ratio of the scintillation energy to the incident particle energy, and sometimes the amount of light output is used to represent the luminous efficiency, which is defined as the ratio of the average number of photons emitted by the scintillation body to the energy absorbed by it.

2) The emission spectra should match the spectral response of the photomultiplier. Not monochromatic light emitted by the scintillator, which wavelength there is a continuous distribution. generally it is of very wide emission spectrum, there are one or several peak, usually in the scintillator characteristics are given corresponding to the main peak wavelength, the wavelength is called the strongest wavelength.. Photocathode of the photomultiplier tube for different wavelength of the photon photoelectric conversion efficiency is different, there is a match with the spectral response of the photomultiplier tube.

3) Luminescence time. the scintillation luminescence time is two part of the rising time and the decay time of the optical pulse. The output pulse width of the scintillation detector is mainly depended on the scintillation luminescence time. Its resolution time is shorter and more suitable for fast counting, and and long scintillator cannot be used as a probe.too long of the scintillation detector can not be used as a probe. The scintillator with too long light emitting time can not be used as scintillation probe.

TABLE I. THE PERFORMANCE OF SOME COMMON SCINTILLATION

\begin{tabular}{|c|c|c|c|c|c|}
\hline Scintillator & $\begin{array}{c}\text { Type of } \\
\text { scintillati } \\
\text { on }\end{array}$ & $\begin{array}{c}\text { Densi } \\
t y(g / 1 \\
\left.0^{-3}\right)\end{array}$ & $\begin{array}{c}\text { Wavelengt } \\
\text { h (nm) }\end{array}$ & $\begin{array}{c}\text { Time } \\
\text { constan } \\
\text { t(ns) }\end{array}$ & $\begin{array}{c}\text { Relative } \\
\text { light } \\
\text { output }\end{array}$ \\
\hline $\mathrm{Nal}(\mathrm{Tl})$ & \multirow{4}{*}{ Organic } & 3.67 & 413 & 230 & 230 \\
\hline $\mathrm{Nal}$ & & 3.78 & 303 & 60 & 440 \\
\hline $\mathrm{Csl}(\mathrm{Tl})$ & & 4.51 & 580 & 1100 & 95 \\
\hline $\mathrm{ZnS}(\mathrm{Ag})$ & & 4.09 & 450 & 800 & 338 \\
\hline Anthracene & \multirow{4}{*}{ Inorganic } & 1.25 & 447 & 30 & 100 \\
\hline Qi & & 1.16 & 410 & 4.5 & 50 \\
\hline Liquid & & $\sim-0.9$ & $380 \sim 420$ & 2.8 & $50 \sim 80$ \\
\hline Plastic & & $\sim 1.0$ & $380 \sim 420$ & $2 \sim 4$ & $50 \sim 70$ \\
\hline
\end{tabular}

In addition, also want to consider the detection efficiency, stopping power, transparency, and other parameters. The issues that need quick count, want to choose a smaller scintillator.See from table 3-1, the scintillation of minimum luminescence time is the plastic scintillator, Its maximum emission wavelength is $380 \sim 420 \mathrm{~nm}$, which is matched with the universal photo multiplier tube.But the relative light output of Nal single crystal and $\mathrm{Nal}(\mathrm{Tl})$ single crystal is larger, while the light emitting time the latter is 100 times of the former.By comparing, since the plastic scintillator emission time is short, in ns, and a high degree of transparency, light transmission performance is good, but also stable radiation performance, high mechanical strength, resistant to moisture, do not need to be encapsulated, the price is low, was chosen as the scintillator. Although it is less than the low efficiency of light emission can be compensated by the subsequent circuit, does not affect the sensitivity of the entire system.

\section{B. Selection of photoelectric multiplier tube}

It is made up of an optical cathode, several dynodes and an anode sealed in a glass tube The function of photocathode is to receive the photons and emit photoelectron, the function of dynodes is to receive the photoelectron and launch more 
electronics, the anode is used to receive electrons and form a voltage pulse at its output.The selected photo multiplier tube should have the following indicators.(1) High photocathode sensitivity.(2)The spectral response of the photocathode is well matched with the spectral characteristics of the scintillation.(3)Large linear range of the photocathode.(4) Background pulse and dark current is small.(5)Electronic flight time is short and the fluctuation is small.(6)The stability and repeatability of each parameter of the photoelectric multiplier tube are good.(7)It can withstand greater flux, and the sensitivity is not changed with the load strong radioactive radiation.(8)Poor sensitivity to electromagnetic fields and strong radioactive radiation.

In this problem, the high count rate requirement is required, Therefore, should choose the focusing photomultiplier tubes with small resolution time.(1)In the cassette containing the scintillator (usually with aluminum, a thickness of about $2 \mathrm{~mm}$ ) under translucent case, the high voltage is not allowed, otherwise it will damage the photoelectric tube, the scintillator exposed, it is not immediately available.(2)In order to reduce the noise, the photoelectric multiplier must work in an absolute dark environment.(3)To improve measurement sensitivity, the dynode voltage should be higher level in the first two, and the rest can take the equivalent, and the last few stages can be connected in parallel with the proper regulators capacitance capacity. $R$ value takes $0.5 \sim 50 \mathrm{M} \Omega$ order of magnitude to save the total current of high voltage power supply, further improve the load capacity, the total current is not less than the maximum of 10 times of the anode current for the design standards to avoid saturation on photocurrent.In addition, the divider resistance should be highly stable.(4)Using magnetic alloy as the magnetic shield, in order to overcome the influence of ionization region in the muzzle. (5)Because if the high voltage power supply has $1 \%$ change, the magnification of the photomultiplier tubes will be a change of about $10 \%$, so the stability demanding for high voltage power supply is very high. Except, in order to improve the optical contacts between the scintillators and photomultiplier tubes, and to increase the collection efficiency of flash, should put some silicone oil with high refractive index (or other material) as coupling agent.

\section{Problems in the application of scintillation detectors}

The scintillation detector has two kinds of working states, average current state and pulse state. This problem can be employed two states, the subsequent processing circuit is simple in the average current operating state, but the sensitivity is low. the subsequent processing circuit is more complex, but the measurement accuracy of the system can be improved in the pulse current operating state, but the measurement accuracy of the system can be improved. Reducing the impact caused by statistical fluctuations, this issue focuses on the pulse operation.The output pulse form of the scintillation detector depends on two factors: the scintillation luminescence decay time and the RC time constant of the output circuit. According to what has been discussed above, it is advisable for the RC to take about $1 / 5$ of the luminescence decay time of scintillation at this time the pulse form mainly by determined by the scintillation luminescence decay time. When measuring $\mathrm{X}$ line source for tube voltage $60 \mathrm{kVp}$ with the plastic scintillation detector, the output pulse amplitude to make a estimate.(1)the Plastic scintillator detector absorbs energy of th X-rays in nearly $60 \mathrm{keV}$, the output pulse amplitude is the greatest of all.(2)Energy conversion efficiency of $6 \%$, or about $3.6 \mathrm{meV}$ energy into light energy,the average energy of the photons emitted by the plastic scintillation body is about $3 \mathrm{eV}$, and thus the emission is about 1200 photons.(3)The photon collection efficiency is about $30 \%$, the photoelectric conversion efficiency of the photocathode is about $10 \%$. As a result, there are about 36 optoelectronic generated.(4)The multiplication factor of Multiplier photomultiplier tubes is 105 to 106 , such as taking $5 \times 105$. The anode can be collected at approximately $1.8 \times$ 107 electrons.(5)Each electron charge is $1.6 \times 10-19 \mathrm{C}$, the total charge collected by the anode is about $2.88 \times 10-12 \mathrm{C}$. Assuming the total distribution capacitance of the output circuit of the photoelectric multiplier is $10 \mathrm{pF}$, and the output pulse amplitude is about $300 \mathrm{mV}$.

The scintillation detector not only there are two ways of signal output, there is also two different high voltage connections on the anode and cathode. To facilitate the processing of the subsequent circuit, and to avoid the high pressure-resisting, adopt the method of the cathode connected negative high voltage and the anode grounded, although it has damaged the focus effect. But on the a cover of photomultiplier tube coupled with the same potential negative high voltage as the cathode, to remove the impact.The count rate of scintillation detector varies with the external high voltage changed.on the detector. Only in a certain range of voltage, the count rate does not change with the external high voltage, that is, there is a flat, see figure 3. And for different amplifier gain, the length of a flat is different.The figure shows that as the gain increases, the slope of a flat is reduced. Therefore, the operating voltage of the tube should normally be selected in the middle or low part of the flat area in order to keep stable work, and also has a low background noise, debugging process should be paid particular attention.

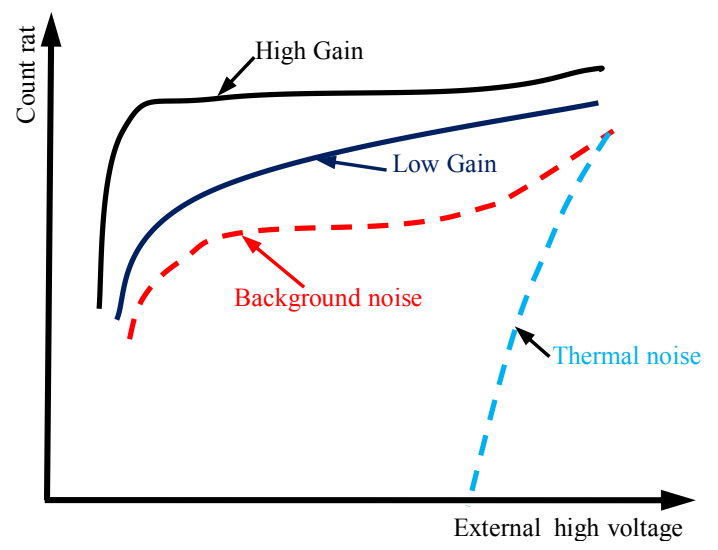

Figure 3. The relationship between the change of the external voltage and the count rate of the plastic scintillation counter 


\section{POWER SUPPLY TO THE DETECTOR}

For the high voltage power supply of the photoelectric multiplier tube, the output voltage depending on the different types of photomultiplier tubes to hundreds of volts to three kilovolts and adjustable, The output current is approximately a few ma. in order to meet the detector output and count rate into a good linear relationship, reduce fluctuation error, so the stability of the high voltage power demand is very high, up to $2 / 10000$.The high voltage power supply of the system is realized by the DC DC converter using transistor. The main unit of the method is an oscillator, low voltage DC to the oscillator power supply, the AC output of the oscillator is boosted by a transformer, and after the rectifier, the filter is used to obtain the DC high voltage. Its block diagram is shown in figure 4 .

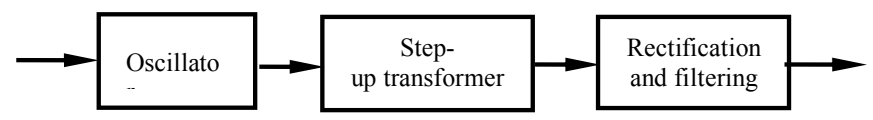

Figure 4. Principle diagram of DC-DC conversion

Using the oscillating circuit is the push-pull double transformer rectangular wave oscillation circuit.This is composed of two transistors coupled by the transformer positive feedback circuit. The loop gain is greater than 1, which meet the condition of oscillation, the working frequency of the oscillator in hundreds of $\mathrm{Hz}$ to dozens of $\mathrm{Hz}$. No large current spikes appear in compared to the oscillation circuit with single transformer structure of push-pull type rectangular wave oscillating circuit, so the reverse voltage overload also reduced to a minimum.

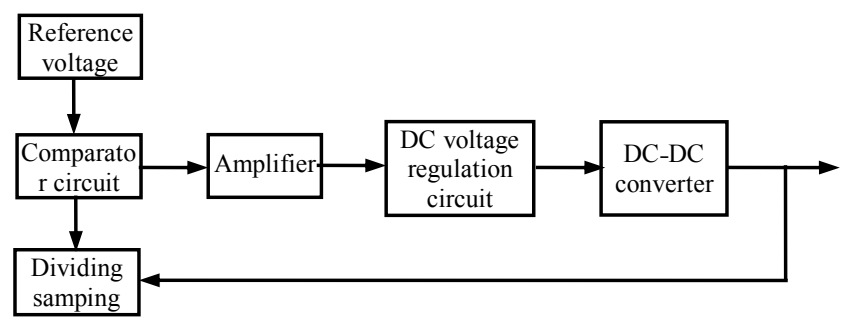

Figure 5. Principle diagram of DC high voltage regulation

DC high voltage obtained by the DC-DC converter is unstable, in order to meet the requirements of the detector to bias voltage, DC voltage must make DC high voltage stable enough, figure 5 is the adjustment of dc high voltage system.to adjust the system Figure 5 DC high voltage. After DC voltage output obtained by the DC-DC converting goes through dividing and sampling, compared with the reference voltage, when the DC voltage output high (low) and an error signal obtained by the comparison, the signal is amplified enough to DC low-voltage regulator circuit,, make the output of the DC low voltage corresponding to lower (or higher), The low-voltage DC supply the oscillator in the DC-DC conversion circuits for the power supply,so that the output voltage of the oscillator is corresponding to lower (or higher), the negative feedback effect in that the DC output voltage stable within a certain range. The stability of Dc high voltage is good or bad, depends on the stability of the reference voltage, high stability of sampling, the stability of the comparison amplifier output and the whole system closed loop amplification. the output voltage of the power supply By changing the number of transformer secondary winding turns to realize sub-file adjustment, to achieve fine adjustment by using the potentiometer.

\section{CONCLUSION}

In the experiment, the capacity of the X-ray source is a voltage $60 \mathrm{KV}$, the current is less than or equal $1 \mathrm{~mA}$, the plastic scintillator detector probe was adopted, the model of the photomultiplier tube is GDB - $44 \mathrm{w}$ as the working voltage $630 \mathrm{v}$, the size of the slit is $20 \mathrm{~mm} \mathrm{X} 0.2 \mathrm{~mm}$, measured weapon is 56 semi-automatic rifles, which this calibre is7.62 $\mathrm{mm}$ the projectile play is $26.5 \mathrm{~mm}$ long, the muzzle velocity is 710 to $725 \mathrm{~m} / \mathrm{s}$, the distance from source to detector is $50 \mathrm{~cm}$. The results show that X-ray will not be affected in the muzzle flame this test method is feasible and effective. The pulse signal capturing from experiment can see, the output signal of the X-ray target detector than the laser target is a more ideal and the better consistency in muzzle area.Because there is no the detector output of the amplification, so the delay will be smaller, and the dynamic sensitivity will be higher. The measure precision of the measurement system can be as high as $0.1 \%$.

\section{ACKNOWLEDGMENT}

The author wishes to thank the Mr. Hao Zhongkao of Department of Information Technologies in comparison test and all colleagues who previously provided technical support.

\section{REFERENCES}

[1] Li Zhe,Tuo Xianguo,Shi Rui,Yang Jianbo, A statistical approach to fit Gaussian part of full-energy peaks from $\mathrm{Si}(\mathrm{PIN})$ and $\mathrm{SDD} \mathrm{X}$-ray spectrometers[J].Science China(Technological Sciences), No.1,pp. 1924, Jan.2014.

[2] Jiang Hua,Zhou Yuanyuan,Research on Structure and Performance of Photomultiplier[J]. Ship Electronic Engineering, No.1,pp. 193-96, Jan.2009.

[3] Ye Lihua,Wang Haiyang etc. Design of Low-Noise Preamplifier on PMT and Its signal Processing[J].Chinese Journal of Electron Devices, No.3,pp. 201-04, Jun.2013.

[4] Wei Yunpeng, Fang Weixin.Factors Influencing Measurement Accuracy of X-ray Thickness Gauge and Appropriate Compensation Measure Process Automation InstrumentationTechnology Application, No.5,pp 79- 82, Oct. 2011.

[5] Fang Jianzhong Yang Jinwen.Application X-ray to Physics Experiment Teaching in Collage Journal of Taiyuan Normal University(Natural Science Edition), No.3,pp 95-98, Feb. 2011.

[6] Jiang Hua, and Zhou Yuanyuan, "Research on Structure and Performance of Photomultiplier". Ship Electronic Engineering, Vol. 29, No.1, pp. 193-96, Jan. 2009

[7] Ye Lihua and Wang Haiyang etc., "Design of Low-Noise Preamplifier on PMT and Its signal Processing". Chinese Journal of Electron Devices, Vol. 36, No.3,pp. 201-04, Jun. 2013.

[8] Xia Jiangtao,Fang Runchen and Yuan Changbin, "Design of HighVoltage Power Supply in Photomultiplier". Modern Electronics Technique, Vol. 33, No.3, pp. 340-42, Jun. 2010.

[9] Zhou Qang, Fan Xaojie, Hu Xin.Receiving and Processing of Shortrang Pulse Laser Detecton.”. Electro-Optic Technology Application, Vol. 27, No.1, pp. 29-33, May. 2012. 